\title{
AERODYNAMIC PRIMARY BREAKUP AT THE SURFACE OF NONTURBULENT ROUND LIQUID JETS IN CROSSFLOW*
}

\author{
J. Mazallon, Z. Dai and G.M. Faeth \\ Department of Aerospace Engineering \\ The University of Michigan \\ Ann Arbor, Michigan 48109-2140
}

\begin{abstract}
$\underline{\text { Abstract }}$
An experimental investigation of nonturbulent round liquid jets in air crossflows at normal temperature and pressure was carried out, using pulsed shadowgraphs to observe jet deformation and breakup. Test liquids included water, ethyl alcohol and glycerol mixtures; liquid jet diameters were in the range $0.8-13 \mathrm{~mm}$; liquid velocities were in the range $0-50 \mathrm{~m} / \mathrm{s}$; and air velocities were in the range $0-24 \mathrm{~m} / \mathrm{s}$. The corresponding test range involved Weber numbers of 2-200, Ohnesorge numbers of 0.00006-0.3, liquid/gas momentum ratios of $100-8000$ and liquid/gas density ratios of 580-1020. The observations suggest qualitative similarities between the surface breakup of nonturbulent round liquid jets in crossflow and the secondary breakup of drops; for example, for Ohnesorge numbers less than 0.1 , the onset of breakup occurs as bag breakup beginning at a Weber number of 5 , there is a second transition to a bag/shear breakup regime at a Weber number of 60 and a third transition to a shear breakup regime at a Weber number of 110 . A long-ligament shear breakup regime appears at Ohnesorge numbers greater than 0.1 . At the onset of breakup, the deformation of the liquid column yields a frontal diameter roughly twice the initial jet diameter, relatively independent of the breakup regime. The characteristics of waves associated with the breakup process were also studied, finding that bag and bag/shear breakup involved both liquid column and surface waves while shear breakup involved only surface waves. The results also showed that breakup characteristics were mainly influenced by the Weber number while effects of the liquid/gas momentum ratio are small.
\end{abstract}

\section{Nomenclature}

$\mathrm{d}_{\mathrm{f}} \quad=$ frontal dimension of the liquid jet

$\mathrm{d}_{\mathrm{inj}} \quad=$ injector diameter

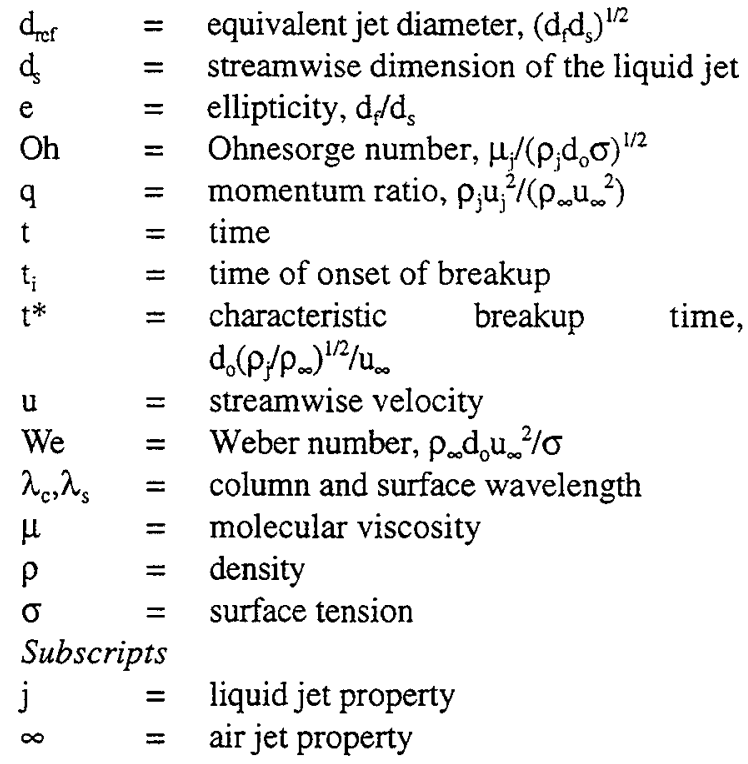

Introduction

The deformation and breakup of nonturbulent round liquid jets in subsonic gaseous crossflows were studied experimentally. The investigation was motivated by applications of spray breakup in crossflow to airbreathing propulsion systems, liquid rocket engine combustion, diesel engines, spark ignition engines and agricultural sprays, among others. The present experimental methods were similar to past studies of primary $^{1-4}$ and secondary ${ }^{5.9}$ breakup in this laboratory.

Earlier studies of round liquid jets in crossflow were recently reviewed by Wu et al. $;^{10}$ therefore, the following discussion of past research is brief. Past research has mainly focused on penetration lengths and jet/spray plume trajectories for various liquid properties, liquid jet properties and crossflow properties. ${ }^{10-22}$ In particular, Wu et al. ${ }^{10}$ report similarities between the breakup properties of round liquid jets in crossflow and the

"Copyright $\odot 1998$ by G.M. Faeth. Published by the American Institute of Aeronautics and Astronautics, Inc., with permission. 
secondary breakup of drops. A limitation of Wu et al. ${ }^{10}$ however, is that jet exit conditions known to affect liquid jet breakup properties, ${ }^{4}$ were not well defined and effects of flow properties on the appearance of particular breakup regimes were not quantified. Earlier studies of the properties of round liquid jets in crossflow ${ }^{11-22}$ had similar limitations.

The objectives of the present investigation were to help fill this gap in the literature by observing the properties of uniform nonturbulent round liquid jets in crossflow, seeking to quantify effects of parameters known to influence processes of both the primary breakup of liquid jets in crossflow and the secondary breakup of drops. Present measurements included pulsed shadowgraph photographs of nonturbulent round liquid jets of various liquids (water, ethyl alcohol and glycerol mixtures) in air crossflows at normal temperature and pressure.

The paper begins with a discussion of experimental methods. Results are then described considering flow visualization, breakup regimes, liquid column deformation, onset of breakup and the properties of waves associated with the breakup process, in turn. The paper ends with a summary of the major conclusions of the study.

\section{Experimental Methods}

\section{Apparatus}

A sketch of the experimental apparatus appears in Fig. 1. The arrangement consisted of a liquid nozzle assembly and an air crossflow assembly. The uniform nonturbulent round liquid jets were injected vertically downward and normal to the air crossflow using a pressure feed system. The test liquid was contained within a cylindrical chamber having either a round sharp-edged nozzle (a Borda nozzle) or a round supercavitating nozzle (having a sharp-edged inlet and exit with a lengthto-diameter ratio less than three), both of which yield uniform nonturbulent round liquid jets as discussed by $\mathrm{Wu}$ et al. ${ }^{4}$ and Leinhard. ${ }^{23}$ Several different Borda nozzles (2, 4 and $16 \mathrm{~mm}$ diameter) and the supercavitating nozzle (1 $\mathrm{mm}$ diameter) were used to provide liquid jets having various diameters (note that actual liquid jet diameters are only $50-70 \%$ of the geometrical diameter of these nozzles, see Lienhard ${ }^{23}$ and Dai et al. ${ }^{24}$ ).
The test liquid was placed within the cylindrical chamber using a liquid feed line (not shown in Fig. 1). A cork in the nozzle passage was used to prevent premature outflow of the liquid. Pressurized air was admitted to the top of the chamber upon activation of a solenoid valve; this air was stored in a large $\left(1.3 \mathrm{~m}^{2}\right.$ volume $)$ air reservoir set to the desired injection pressure by filling from the laboratory high-pressure air supply system (pressures up to $1500 \mathrm{kPa}$ with a dew point less than $240 \mathrm{~K}$ ). The increased pressure in the cylindrical chamber due to air inflow caused liquid outflow once the cork was forced out of the nozzle. Excessive aeration of the test liquid during injection was prevented by a baffle across the air inlet of the cylindrical chamber. Once all the liquid was forced out of the liquid chamber, the solenoid valve was closed and the chamber was allowed to vent to the surrounding atmosphere. The cork was then replaced and the chamber was refilled for the next test. The pressure of the air in the air reservoir was varied to provide liquid velocities at the nozzle exit in the range $0.50 \mathrm{~m} / \mathrm{s}$. The corresponding liquid injection times were as small as $200 \mathrm{~ms}$, however, this was not a problem because flow development times were quite short (less than 10\% of the liquid injection times) while data acquisition times using pulsed shadowgraph photography were even shorter (requiring less than $10 \mu \mathrm{s}$ ).

The air crossflow was provided by a blower drawing air at normal temperature and pressure from the laboratory. The blower flow was regulated using a gate valve (not shown in Fig. 1) to provide mean crossflow velocities in the range 0 $24 \mathrm{~m} / \mathrm{s}$; these velocities were measured using a vane anemometer (Omega, Model HH-31). The flow leaving the blower passed through a honeycomb section (hexagonal cells $8 \mathrm{~mm}$ wide having a length of $50 \mathrm{~mm}$ ) and three screens (wire diameters of $0.3 \mathrm{~mm}$ in a square pattern on $1 \mathrm{~mm}$ centers) to yield a relatively uniform nonturbulent and nonswirling horizontal flow having a diameter of $200 \mathrm{~mm}$. The outlets of the liquid jet nozzles were placed $20 \mathrm{~mm}$ above the upper edge of the uniform region of the air crossflow. Distributions of streamwise mean and fluctuating crossflow velocities were measured using a hot-wire probe (TSI digital monitor and power supply, Model 1051-1, constant-temperature linearized anemometer, Model 1054A, variable decade probe resistance, Model 1056, and sensor probe Model 1210-60). These observations indicated relatively uniform streamwise mean velocities (within 5\%) 
and r.m.s. streamwise velocity fluctuations of less than $2 \%$ over the crossflow.

The nozzle and crossflow assemblies were connected to form a single rigid unit. This unit was then placed on a mounting frame that could be adjusted so that the assembly could be positioned in the vertical direction to accommodate rigidlymounted optical instrumentation. The deflected liquid jet was directed downward into a collecting tank using a baffle (neither are shown in Fig. 1). The test liquid was discarded after use in order to maintain liquid cleanliness. The entire apparatus was contained within a $2100 \times 1100 \times 660 \mathrm{~mm}$ plastic enclosure with windows for optical access, in order to control liquid splashing.

Instrumentation

The present measurements were limited to pulsed shadowgraphy. Light sources consisted of either a xenon arc lamp (Xenon Corp. Model 437Bnanopulser) that generated a light pulse having an optical energy up to $10 \mathrm{~mJ}$ and a duration of 20 ns, or a frequency-doubled YAG laser (Spectra Physics, Model GCR-130) yielding a light pulse having an optical energy up to $300 \mathrm{~mJ}$ and a pulse duration of $7 \mathrm{~ns}$. The light pulses from both these sources were short enough to stop the motion of the flow. The light beams were expanded to provide collimated beams having diameters of $30-60 \mathrm{~mm}$ through the flow.

The shadowgraph images were recorded using a $100 \times 125 \mathrm{~mm}(4 \times 5$ inch $)$ Graphlex still camera. The camera was focused at the plane of symmetry of the deflecting liquid jet with the image magnified 2-3:1 on the film. The camera was operated with an open shutter within a darkened room so that exposure times were controlled by the duration of the light pulses. The images were recorded using Polaroid Type 55-57 black and white film.

One property of interest was the crosstream (frontal) and streamwise dimensions of the liquid jets. Unfortunately, the present test arrangement could not provide simple projections of both these images; instead, two cameras were used to record images of the liquid jets along optical axes that were horizontal and were directed at angles of $48^{\circ}$ and $90^{\circ}$ to the direction of the crossflow. The resulting images were then analyzed assuming that the liquid column had an elliptical cross-section, similar to the approach used by Nguyen and Karagozian. ${ }^{20}$

\section{$\underline{\text { Test Conditions }}$}

The test liquids included water, ethyl alcohol and glycerol mixtures $(66,79$ and $84 \%$ glycerin by mass in water). The properties of the test liquids are summarized in Table 1. The liquid properties summarized in Table 1 were measured as follows: liquid density using a set of hydrometers (Fisher, Model 11-582), liquid viscosity using a Cannon-Fenske viscosimeter (Fisher, Model 13617 ) and surface tension using a ring tensiometer (Fisher, Model 20). The present results for pure liquids agreed with the values given by Lange ${ }^{25}$ within experimental uncertainties.

Test conditions were varied by considering the various liquids, liquid jet diameters of $0.8-13$ $\mathrm{mm}$, liquid jet velocities of $0-50 \mathrm{~m} / \mathrm{s}$ and air crossflow velocities of $0-24 \mathrm{~m} / \mathrm{s}$ at normal temperature and pressure. This yielded the following ranges of normalized test variables: the ratio of drag-to-surface tension forces, characterized by the Weber number, We, in the range 2-200; the ratio of liquid viscous-to-surface tension forces, characterized by the Ohnesorge number, $\mathrm{Oh}$, in the range $0.00006-0.3$; the ratio of liquid-to-gas momentum fluxes , $\mathrm{q}$, of 100-8000; and the ratio of liquid-to-gas densities, $\rho_{j} / \rho_{\infty}$, of 680-1020.

\section{Results and Discussion}

\section{Flow Visualization}

The presentation of the results will begin with pulsed shadowgraph photography for flow visualization of various liquid jet breakup conditions. The properties of the injection process of the present nonturbulent round liquid jets in still air are illustrated by the photograph of Fig. 2 . These test conditions involved a water jet with a liquid jet diameter of $1.1 \mathrm{~mm}$, using a Borda nozzle having a diameter of $2 \mathrm{~mm}$, but the appearance of this jet is typical of present liquid jets in the absence of crossflow. It is evident that the surface of the liquid column is smooth and that no disturbances or protrusions are observable over the $30 \mathrm{~mm}$ vertical height of the image. In fact, this behavior persisted over the observable length of the liquid jet which was up to $300 \mathrm{~mm}$ long. Thus, 
present behavior for uniform nonturbulent jet exit conditions was very similar to a liquid cutting jet, which is consistent with past observations of the behavior of uniform nonturbulent round liquid jets, as well as nonturbulent plane wall jets, for injection into still gases with liquid/gas density ratios greater than $500^{4,23,24}$ These results also provide direct proof that all breakup processes observed during the present investigation were caused by the air crossflow rather than by liquid vorticity or turbulence that leads to breakup processes such as turbulent primary breakup. ${ }^{2.3}$

For conditions where effects of liquid viscosity were small $(\mathrm{Oh}<0.1)$ five kinds of flow were observed as the crossflow velocity (characterized by the Weber number) was increased: simple, deformation of the shape and trajectory of the liquid jet with no breakup, breakup of the liquid column as a whole, bag breakup, bag/shear breakup and shear breakup. The general appearance of the breakup of nonturbulent round liquid jets in crossflow can be seen from the composite pulsed shadowgraph photography illustrated in Fig. 3. These conditions were within the bag breakup regime near its transition from the column breakup regime. For these conditions, the column first deforms in the direction normal to the crossflow, to yield an ellipsoidal cross-section. This behavior is caused by the reduced gas pressure near the side of the jet as the gas accelerates over the liquid column, with the resulting lateral motion of the liquid stabilized to some extent by surface tension, somewhat analogous to the behavior of individual drops subjected to shock wave disturbances in the deformation regime. ${ }^{5.7}$ The increased drag forces due to the flattened shape of the column also enhance the tendency of the liquid column to be deflected downstream with respect to the gas motion. Once the jet has flattened sufficiently, and has started to deflect in the downstream direction, bag-like structures appear which are very similar to the bag breakup regime observed during the secondary breakup of drops. ${ }^{5.6}$ This behavior involves the formation of bags as a result of the deformation of the central portion of the liquid column downstream due to the higher pressure of the stagnating gas flow on the upstream side of the flattened liquid column. The development of bags is not a twodimensional phenomenon, however, due to the appearance of nodes along the liquid column, characterized by a wavelength of separation between the nodes of $\lambda_{c}$. With increasing distance along the liquid column, the bags first progressively grow in the downstream direction. As the bags approach their maximum size, however, they begin to progressively break up beginning at their tip. This process appears to be the result of Rayleigh-like breakup of the thin liquid sheet, augmented by motion of the liquid/gas edge of the bag due to unbalanced surface tension forces, very similar to the bag breakup process of drops. ${ }^{10}$ Upon completion of the breakup of the bags themselves, two strings of node drops, connected by thinner liquid columns that deflect downstream due to the drag forces caused by the crossflow. The connecting liquid columns then break up, probably due to Rayleigh breakup in a manner similar to the basal ring of secondary drop breakup in the bag breakup regime. ${ }^{5.6}$ The final result is a polydisperse array of large drops associated with the presence of nodes and the breakup of their connecting liquid columns, along with a large number of much smaller drops associated with breakup of the bags themselves. This behavior tends to separate drops according to size along the deforming liquid column, with the smallest drops appearing near the onset of breakup and drop sizes progressively increasing with increasing distance along the liquid jets.

The appearance of the four liquid jet breakup regimes at $\mathrm{Oh}<0.1$ will now be considered in the order of progressively stronger gas disturbances, i.e., increasing Weber numbers.

The breakup mechanism observed at the smallest crossflow velocities used during the present experiments was liquid column breakup as a whole. A pulsed shadowgraph photograph of typical breakup behavior in this regime is illustrated in Fig. 4. The liquid-column breakup mode involves deflection of the liquid column as a whole in the streamwise direction of the crossflow, distortion of the column into a flattened shape and the appearance of somewhat thickened regions along the column. The thickened regions (nodes) subsequently develop into drop-like structures with thinner interconnecting liquid column regions that deflect in the streamwise direction compared to the slower moving node drops. Breakup of the liquid column then finally occurs by breakup of the thin liquid column regions due to Rayleigh-like breakup, or Rayleigh-like breakup supplemented by stretching of the thin liquid columns. Naturally, very low crossflow velocities would eventually lead to transition from this breakup regime to a stable liquid jet similar to Fig. 2. It was not possible to find the transition to the liquid column breakup 
regime, however, due to the limited dimensions of the present crossflow.

As crossflow velocities increase, the next breakup regime observed is the bag-breakup regime. One illustration of the bag-breakup regime has already been discussed in connection with Fig. 3; another illustration of this breakup regime appears in Fig. 5. The bag-breakup regime involves deflection of the liquid column in the streamwise direction of the crossflow, the appearance of wavelike deformation of the liquid column and the appearance of nodes, similar to the liquid column breakup regime. In this case, however, the thin liquid columns between nodes and the nodes themselves split apart, allowing bag-like structures to grow with the wavelengths of the bag-like structures similar to those of the nodes. The baglike structures then break up as described in connection with Fig. 3 so that the liquid flow develops into two liquid columns of wavelike structures involving node drops and interconnecting liquid columns. Finally, each of these liquid columns undergoes liquid column breakup. The final outcome involves polydisperse population of drops whose mean sizes progressively increase with increasing distance along the liquid jet. The outcome of breakup includes the small drops formed by breakup of the bags, larger drops formed by breakup of the liquid columns between node drops and the largest drops formed from the node drops. As noted earlier, this general behavior bears many similarities to the secondary breakup of drops in the bag breakup regime, see Refs. 5-9 and references cited therein.

Further increases of crossflow velocities cause transition to a complex breakup regime that is a combination of bag breakup that was just discussed and shear breakup that is observed at the largest crossflow velocities. An illustration of typical behavior in this bag/shear breakup regime appears in Fig. 6. In this case, both bag-like structures due to penetration of the liquid column, and liquid ligaments due to the shearing of liquid from the periphery of the liquid column, appear at roughly the same time. Breakup then involves breakup of the bag-like structures as discussed in connection with Fig. 5 as well as breakup of the ligaments in a manner that appears to be very similar to the secondary breakup of drops in the shear breakup regime, see Refs. 5-9 and references cited therein.
The largest crossflow velocities considered during the present investigation cause transition to the shear-breakup regime. A pulsed shadowgraph photograph of typical behavior in this regime is illustrated in Fig. 7. Similar to the bag and bag/shear breakup regimes, the process begins by deflection of the liquid column in the streamwise direction of the crossflow followed by distortion of column into a somewhat flattened shape. Wavelike disturbances also appear in the upstream side of the liquid column but the wavelengths of these disturbances are smaller than the diameter of the liquid column and they do not develop into the nodes observed in the liquid-column and bagbreakup regimes. Instead, ligaments are stripped from the periphery of the liquid column, very similar to the behavior of secondary drop breakup in the shear breakup regime, see Refs. 5-9 and references cited therein. Within the ligament structures, occasional sheet-like regions are observed, however, these regions appear to be sheets between ligaments rather than bags.

As discussed subsequently, effects of liquid viscosity, characterized by the Ohnesorge number, were not very significant for $\mathrm{Oh}<0.1$. Within the shear breakup regime, however, the length of the ligaments being stripped from the sides of the liquid column progressively increased with increasing Ohnesorge number. Thus, similar to the secondary breakup of drops in the shear breakup regime, ${ }^{18}$ a long-ligament shear breakup regime was defined for $\mathrm{Oh}>0.1$. A pulsed shadowgraph photograph of the typical appearance of the region near the liquid column as the long-ligament regime is approached appears in Fig. 8. The long ligaments are clearly evident; in addition, there are thin liquid sheets between the ligaments similar to the appearance of the flow in Fig. 7. The longligament regime presents significant experimental difficulties due to the problem of tracking flow behavior when ligaments are long; therefore, the present measurements generally were limited to Ohnesorge numbers smaller than the long-ligament regime.

\section{Breakup Regimes}

Exploiting the similarities between the breakup regimes of nonturbulent round liquid jets (liquid jets) in crossflow, and the secondary breakup of drops, the liquid jet breakup regimes were correlated in terms of the Weber and Ohnesorge 
numbers as first proposed by Hinze ${ }^{26}$ for the secondary breakup of drops exposed to shock-wave disturbances and subsequently used by most investigators of secondary drop breakup, see Refs. 5-9 and references cited therein. The resulting breakup regime map is plotted in Fig. 9. For present conditions (We of 2-200 and $\mathrm{Oh}$ of $0.00006-0.3$ ) the liquid jet breakup regimes are relatively independent of $\mathrm{Oh}$, with four breakup regimes identified as discussed in connection with the flow visualization study, as follows: liquidcolumn breakup (We $<5$ ), bag breakup $(5<\mathrm{We}<$ 60 ), bag/shear breakup $(60<\mathrm{We}<110)$ and shear breakup $(110<W e)$. Finally, it should be noted that variations of liquid/gas momentum and density ratios did not have a significant effect on these breakup regime transitions over the present test range, i.e., $q$ in the range $100-8000$ and $\rho_{j} / \rho_{\infty}$ in the range 680-1020. The four liquid jet breakup regimes plotted in Fig. 9 are somewhat analogous to the secondary breakup regimes of drops subjected to shock-wave disturbances. In order to show this relationship, the secondary breakup regime map for drops from Hsiang and Faeth ${ }^{5,6}$ is plotted on Fig. 9 for comparison with the present liquid jet breakup results. The main differences between liquid column and drop breakup are that secondary breakup of drops does not exhibit behavior analogous to liquid column breakup and is only characterized by a drop deformation regime prior to transition to the bagbreakup regime, and that secondary drop breakup responds to a greater degree to increasing $\mathrm{Oh}$ than liquid jet breakup, with progressively increasing We with increasing $\mathrm{Oh}$ at the transitions to various breakup regimes for $\mathrm{Oh}>0.01$. At small $\mathrm{Oh}<$ 0.01 , however, the transitions to secondary drop breakup regimes become relatively independent of Oh, similar to the liquid jet breakup and have the following values at the transitions: deformation (We $<13$ ), bag breakup $(13<$ We $<35)$, multimode breakup $(35<\mathrm{We}<80)$ and shear breakup $(80<\mathrm{We})$. With respect to these transitions, it should be noted that bag/shear breakup of liquid jets is analogous to multimode breakup of drops, and that the original diameters of the liquid jets and the drops should be used in the corresponding expressions for We and $\mathrm{Oh}$.

\section{Liquid Column Deformation}

The first stage of liquid jet breakup involves deformation of the liquid column; this behavior is similar to the secondary breakup of drops where distortion of the drop into a flattened shape invariably precedes drop breakup. The important properties of liquid jet deformation are as follows: the frontal dimension of the liquid jet, $d_{f}$, which is the local width of the jet in the direction normal to the motion of the air crossflow; the streamwise dimension of the liquid jet, $\mathrm{d}_{s}$, which is the local projected length of a jet horizontal jet cross-section in the same direction as the crossflow; the equivalent jet diameter, $d_{\text {ref }}$, which is the geometric mean of these two dimensions, and the ellipticity, which is defined as the ratio of the frontal and streamwise dimensions of the jet.

Typical measurements of the deformation properties of a nonturbulent round liquid jet in crossflow are plotted in Fig. 10. This test condition involves relatively small Weber and Ohnesorge numbers, $\mathrm{We}=7.8$ and $\mathrm{Oh}=0.0012$, with liquid column properties, $\mathrm{d}_{\mathrm{f}} / \mathrm{d}_{\mathrm{ref}}, \mathrm{d}_{\mathrm{s}} / \mathrm{d}_{\mathrm{ref}} . \mathrm{d}_{\mathrm{ref}} / \mathrm{d}_{0}, \mathrm{~d}_{\mathrm{f}} / \mathrm{d}_{\mathrm{o}}$ and e, plotted as a function of normalized crosstream distance, $y / d_{\text {inj. }}$. The first thing to note is that $\mathrm{d}_{\mathrm{Tef}} / \mathrm{d}_{\mathrm{o}}$, approximates unity throughout the deformation process, which implies that the cross-sectional area of the liquid jet is conserved. Such behavior is reasonable because liquid jet velocities do not vary significantly prior to breakup so that the crosssectional area of the liquid jet must be conserved. In contrast, $\mathrm{d}_{\mathrm{f}} / \mathrm{d}_{\mathrm{ref}}$, and $\mathrm{d}_{\mathrm{s}} / \mathrm{d}_{\mathrm{ref}}$ exhibit strong distortion of the jet, with the lateral dimension increasing by roughly $40 \%$. This distortion is also reflected by the large values of ellipticity reached during the process, reaching $\mathrm{e} \sim 3$ for the range of conditions considered in Fig. 10. This large degree of distortion is responsible for the large increases of drag forces and drag coefficients of liquid jets as they break up, seen by Wu et al., ${ }^{10}$ analogous to effects of drop deformation during secondary drop breakup. $^{5-7}$

All the properties illustrated in Fig. 10 continue to increase with increasing $y / d_{i n j}$ over the range of the measurements. This occurs because the jet does not deflect very much as a whole in the region of observations due to the relatively large liquid jet momentum flux of the test condition, i.e., $q=1590$. Results for smaller values of $q$, however, yield much larger deflections of the liquid jet as a whole. Then as the jet becomes more aligned with the crossflow, the tendency of the gas flow to distort the jet decreases and parameters such as $d_{f} / d_{o}, d_{s} / d_{o}$, e, etc., begin to approach unity once again. This behavior is somewhat analogous to the deformation of individual drops due to shock-wave 
disturbances where the deformation regime (at We smaller than the bag-breakup regime) involves the degree of deformation increasing at first and then decreasing once again as the drop continues to accelerate toward the gas velocity.

Another feature of liquid jet breakup in crossflow that is similar to the secondary breakup of drops subject to shock-wave disturbances, is the relative universality of liquid column deformation at the onset of breakup. This behavior is illustrated in Fig. 11 where $d_{f} / d_{0}$ at the onset of breakup is plotted as a function of We for We $>5$ which represents the onset of breakup. It is evident that $d_{f} / d_{0} \approx 1.8$ for We in the range 5-200 for various liquids and values of liquid jet velocity (or $q$ ). For comparison, $\mathrm{d}_{\mathrm{f}} / \mathrm{d}_{\mathrm{o}} \approx 2.0$ at the onset of secondary drop breakup for shock-wave disturbances over a very broad range of Weber numbers, We in the range $13-6000$, at small $\mathrm{Oh}$ (i.e., $\mathrm{Oh}<0.1$ ). ${ }^{5,6}$

\section{Onset of Breakup}

Among all the similarities between liquid jet breakup in crossflow and secondary breakup of drops subjected to shock-wave disturbances, however, is the striking differences between the correlation for the time of onset of breakup. For the secondary breakup of drops, the time of onset of secondary breakup, normalized by the characteristic secondary breakup time of Ranger and Nicholls, ${ }^{27}$ is $\mathrm{t}_{\mathrm{i}} / \mathrm{t}^{*}=2$ for We ranging over the bag, multimode and shear breakup regimes. ${ }^{8.9}$ This behavior can be rationalized by noting that the characteristic velocity within the liquid phase, based on conservation of momentum principles, is $\left(\rho_{\infty} / \rho_{j}\right)^{1 / 2} u_{\infty}$ while it is reasonable to assume that liquid motion must cause deformation of the drop involving a fixed fraction of the initial drop diameter at the time of onset of breakup (since $d_{d} / d_{0}$ is a constant at the onset of breakup for a wide range of $\mathrm{We}$ ). Then assuming that the time required to reach the onset of breakup is proportional to the time required to move a distance equal to the drop diameter, at the characteristic liquid-phase velocity, implies that $t_{i} \sim t^{*}=d_{0}\left(\rho_{j} / \rho_{\infty}\right)^{1 / 2} / u_{\infty}$ or $t_{i} / t^{*}=$ const. as observed for the secondary breakup of drops.

Based on the previous discussion, it seems plausible that $\mathrm{t}_{\mathrm{i}} / \mathrm{t}$ * should also be a constant for the onset of breakup of liquid jets because the characteristic liquid phase velocity is similar to the value for drops while the degree of deformation at the onset of breakup for liquid jets is a constant from the results of Fig. 11, which is also similar to the behavior of drops during secondary breakup. This idea is evaluated based on the results plotted in Fig. 12, where $t_{i}$ is found from the position where the onset of breakup is observed given the known liquid jet velocity. For comparison, past measurements of the times of onset and end of secondary drop breakup are also shown on the plot, based on the results of Ref. 9 and references cited therein. The values of $t_{i} / t^{*}$ for the two types of breakup are of the same order of magnitude but $t_{i} / t^{*}$ for the onset of liquid jet breakup clearly decreases with increasing We, rather than being independent of We similar to the behavior of the secondary breakup of drops. In fact, correlation of the present measurements for $\mathrm{Oh}<0.1$ yields

$$
\mathrm{t}_{\mathrm{j}} / \mathrm{t}^{*}=8.76 \mathrm{We} \mathrm{e}^{-0.62}
$$

with a correlation coefficient of the fit of 0.93 . The corresponding powers of $\mathrm{Oh}(-0.01)$ and $\mathrm{q}(-0.18)$ for a three-variable correlation are relatively small so that it is concluded that Weber number mainly influences $t_{i} / t^{*}$ over the present test range. At the present stage of understanding, liquid jet breakup, however, the reasons for the difference between the behavior of the time of onset of breakup of liquid jets in crossflow and the secondary breakup of drops are not known.

\section{Column and Surface Waves}

Another interesting feature of liquid jet breakup in crossflow is the appearance of waves in the streamwise direction along the liquid column. Two kinds of waves are observed: the wavelengths between nodes, $\lambda_{c}$, involving deflection of the entire liquid column, that have already been discussed in connection with Figs. 3-6; and the smaller wavelength, $\lambda_{s}$, associated with periodic disturbances of liquid stripping along the sides of the liquid column, that already have been mentioned in connection with Figs. 6 and 7.

The appearance of liquid column waves can be seen from the pulsed shadowgraph photograph of Fig. 13. This condition involves a liquid jet having small liquid viscous effects, $\mathrm{Oh}=$ 0.0103 in the liquid column breakup regime close to the transition to the bag-breakup regime at $\mathrm{We}=$ 4.0. The region shown corresponds to the onset of growing liquid column waves. It is seen that the 
amplitude of these waves grows with increasing distance along the liquid column but that the wavelength does not change significantly, i.e., the disturbance appears to convect along the liquid jet. The cusps of these waves eventually are associated with the nodes along the liquid column that are a dominant feature of the bag and bag/shear breakup regimes. It seems likely that these waves are caused by Rayleigh/Taylor instability due to the acceleration of the dense liquid column by the low density gas; in particular, the node structures tend to lag the motion of the rest of the column.

The liquid column disturbances progressively decrease with increasing Weber number as discussed later; in addition, liquid surface waves appear along the liquid column at larger values of We. The appearance of combined liquid column and liquid surface waves can be seen from the pulsed shadowgraph photograph of Fig. 14. This condition involves a liquid jet having small liquid viscous effects, $\mathrm{Oh}=0.0687$ in the bag breakup regime, $\mathrm{We}=8.8$. The region shown corresponds to the onset of growing liquid column and surface waves as well as the onset of bag breakup. The liquid surface waves appear first, they have relatively short wavelengths and they are most prominent on the upstream side of the liquid column. This behavior suggests that they are associated with Rayleigh/Taylor instability similar to the liquid column waves but involve a local phenomena that is not affected by the dimensions of the liquid column itself. With increasing distance along the liquid jet, however, larger wavelength liquid column disturbances appear which eventually dominate the breakup process for these bag breakup conditions.

Normalized liquid column and liquid surface wavelengths, $\lambda_{c} / \mathrm{d}_{\mathrm{o}}$ and $\lambda_{s} / \mathrm{d}_{\mathrm{o}}$ are plotted as a function of the Weber number in Fig. 15. For reference purposes, values of We at transitions to various breakup regimes are also marked on the plots. As noted earlier, liquid column waves first appear in the liquid column breakup regime and eventually are associated with the disturbances that lead to breakup of the liquid column itself as illustrated in Fig. 4. These disturbances begin with $\lambda_{\mathrm{c}} / \mathrm{d}_{\mathrm{o}} \approx 10$ at $\mathrm{We} \approx 1$ and then decrease to $\lambda_{\mathrm{c}} / \mathrm{d}_{\mathrm{o}} \approx 1$ at $\mathrm{We}=60$, which represents the onset of the bag/shear breakup regime. These disturbances decrease at larger We where the breakup process becomes dominated by shear breakup along the sides of the jet which involves more rapidly developing surface waves. The surface disturbance wavelengths are smaller than the liquid column waves, with $\lambda_{s} / d_{0}$ generally less than unity. The wavelength of the surface waves also decreases as We increases and they are still visible in the shear breakup regime where they are associated with the distance between ligaments being stripped from the sides of the liquid columns.

Correlations of $\lambda_{c} / d_{0}$ and $\lambda_{s} / d_{0}$ were sought in terms of $\mathrm{We}, \mathrm{Oh}$ and $\mathrm{q}$. The best fit correlations were achievable as a function of We alone, as follows:

$$
\lambda_{c} / \mathrm{d}_{0}=16.3 \mathrm{We}^{-0.79}
$$

with a correlation coefficient of this fit of 0.86 , and

$$
\lambda_{\mathrm{s}} / \mathrm{d}_{0}=2.82 \mathrm{We}-0.45
$$

with a correlation coefficient of this fit of 0.82 . The effect of Oh on $\lambda_{c} / d_{0}$ and $\lambda_{s} / d_{0}$ could not be resolved within experimental uncertainties while the corresponding powers of $q$ in combined We, $q$ correlations were -0.13 and -0.09 , respectively. Thus, it is concluded that these wavelengths are mainly functions of We over the present test range. Due to the obvious relationships between $\lambda_{c}$ and $\lambda_{s}$ and the properties of bag, bag/shear and shear breakup of liquid jets in crossflow, this primary dependence of breakup properties on We helps strengthen the analogy between the breakup properties of liquid jets in crossflow and the secondary breakup properties of drops subjected to shock-wave disturbances.

\section{Conclusions}

Results of an investigation of nonturbulent round liquid jets in air crossflows at normal temperature and pressure have been described. Test liquids included water, ethyl alcohol and glycerol mixtures; liquid jet diameters were in the range $0.8-13 \mathrm{~mm}$; liquid jet velocities were in the range $0-50 \mathrm{~m} / \mathrm{s}$; and air crossflow velocities were in the range $0-24 \mathrm{~m} / \mathrm{s}$. The corresponding test range involved Weber numbers of 2-200, Ohnesorge numbers of $0.00006-0.3$, liquid/gas momentum ratios of 100-2000 and liquid/gas density ratios of 680-1020. The major conclusions of the study are as follows: 
1. There is a useful general analogy between the surface breakup of nonturbulent round liquid jets in crossflow and the secondary breakup of individual drops which suggests modest streamwise interactions between cross-sections in the jets, i.e., liquid surface deformation and breakup properties are not strongly affected by the liquid/gas momentum ratio for values less than 8000 , the largest value considered during the present study.

2. Transitions to various breakup regimes are not influenced significantly by liquid viscosities for Oh $<0.1$. For these conditions, the onset of breakup occurs as bag breakup at $\mathrm{We}=5$, there is a transition to bag/shear breakup at $\mathrm{We}=60$ and another transition to shear breakup at $\mathrm{We}=110 \mathrm{~A}$ long-ligament shear breakup regime also appears for $\mathrm{Oh}>0.1$.

3. The deformation of the liquid jet prior to the onset of breakup also is somewhat analogous to drop deformation prior to secondary breakup of drops, with the liquid column attaining a frontal diameter roughly twice the initial jet diameter at the onset of breakup, relatively independent of the breakup regime, We, Oh and $\mathrm{q}$.

4. An interesting difference between breakup of nonturbulent round liquid jets in crossflow and the secondary breakup of drops is that normalized time at the onset of breakup, $t_{i} / t^{*}$, is proportional to $\mathrm{We} \mathrm{e}^{-0.62}$ instead of being relatively independent of We similar to the secondary breakup of drops. Values of $t_{\mathrm{i}} / \mathrm{t}^{*}$ were relatively independent of $\mathrm{Oh}$ and $q$, however, for the present test range.

5. The breakup process involved the formation of two kinds of waves: waves involving deformation of the entire liquid column and waves associated with disturbances of the liquid surface. The wavelengths of both types of waves decreased with increasing We but were relatively independent of $\mathrm{Oh}$ and $\mathrm{q}$. The bag and bag/shear breakup regimes involved the presence of both types of waves but in the shear breakup regime only surface waves were observed. An interesting feature of the present observations is that the wavelengths of the column waves are roughly equal to the initial diameter of the jet when the onset of shear-breakup regime is reached.

It should be noted that the present results are limited to nonturbulent liquid jets at relatively large liquid/gas density ratios $\left(\rho_{j} / \rho_{\infty}>500\right)$.
Smaller liquid/gas density ratios and the presence of liquid turbulence are likely to change liquid breakup behavior significantly, based on past observations of both primary breakup of liquid jets and secondary breakup of drops.

\section{Acknowledgments}

This research was supported by the Renault Research Division, Rueil Malmaison, France, under the technical management of $D$. Lahalle and T. Mantel. The support of the Air Force Office of Scientific Research, Grant Nos. F49620-92-J-0399 and F49620-95-I-0364, under the technical management of J.H. Tishkoff, for initial development of the instrumentation is also gratefully acknowledged. The U.S. government is authorized to reproduce and distribute this article for governmental purposes notwithstanding any copyright statement marked hereon.

\section{$\underline{\text { References }}$}

${ }^{1}$ Wu, P.-K., Tseng, L.-K., and Faeth, G.M., "Primary Breakup in Gas/Liquid Mixing Layers for Turbulent Liquids," Atom. Sprays, Vol. 2, 1992, pp. 295-317.

${ }^{2} \mathrm{Wu}$, P.-K., and Faeth, G.M., "Aerodynamic Effects on Primary Breakup of Turbulent Liquids," Atom. Sprays, Vol. 3, 1993, pp. 265-289.

${ }^{3}$ Wu, P.-K., and Faeth, G.M., "Onset and End of Drop Formation Along the Surface of Turbulent Liquid Jets in Still Gases," Phys. Fluids A, Vol. 7, No. 11, 1995, pp. 2915-2917.

${ }^{4}$ Wu, P.-K., Miranda, R.F., and Faeth, G.M., "Effects of Initial Flow Conditions on Primary Breakup of Nonturbulent and Turbulent Round Liquid Jets," Atom. Sprays, Vol. 5, No. 2, 1995 , pp. 175-196.

${ }^{5}$ Hsiang, L.-P., and Faeth, G.M., "Near-Limit Drop Deformation and Secondary Breakup," Int. J. Multiphase Flow, Vol. 18, No. 5, 1992, pp. 635652 .

${ }^{6}$ Hsiang, L.-P., and Faeth, G.M., "Drop Properties After Secondary Breakup," Int. J. Multiphase Flow, Vol. 19, No. 5, 1993, pp. 721735. 
'Hsiang,L.-P., and Faeth, G.M., "Drop Deformation and Breakup due to Shock Wave and Steady Disturbances," Int. J. Multiphase Flow, Vol. 21, No. 4, 1995, pp. 545-560.

${ }^{8}$ Chou, W.-H., Hsiang, L.-P., and Faeth, G.M., "Temporal Properties of Secondary Drop Breakup in the Bag Breakup Regime,"-Int. J. Multiphase Flow, Vol. 23, No. 4, 1997, pp. 651669.

${ }^{9}$ Chou, W.-H., Dai, Z., and Faeth, G.M., "Dynamics of Drop Deformation and Formation During Secondary Breakup in the Bag Breakup Regime," AIAA Paper No. 97-0397, 1997.

${ }^{10}$ Wu, P.-K., Kirkendall, K.A., Fuller, R.P., and Nejad, A.S., "Breakup Processes of Liquid Jets in Subsonic Crossflows," AIAA J., Vol. 13, No. 1, 1997, pp. 64-73.

${ }^{11}$ Geery, E.L., and Margettes, M.J., "Penetration of a High Velocity Gas Stream by a Water Jet," J. Spacecraft, Vol. 6, No. 1, 1969, pp. 79-81.

${ }^{12}$ Reichenbach, R.E., and Horn, K.P., "Investigation of Injectant Properties in Jet Penetration in a Supersonic Stream," AIAA J., Vol. 9, No. 3, 1971, pp. 469-472 .

${ }^{13}$ Kush, E.A., and Schetz, J.A., "Liquid Jet Injection into a Supersonic Flow," AIAA J., Vol. 11, No. 9, 1973, pp. 1223-1224.

${ }^{14}$ Schetz, J.A., and Padhye, A., "Penetration of a Liquid Jet in Subsonic Airstreams," AIAA J., Vol. 15, No. 10, 1977, pp. 1385-1390.

${ }^{15}$ Schetz, J.A., Kush, E.A., and Joshi, P.B., "Wave Phenomena in Liquid Jet Breakup in a Supersonic Crossflow," AIAA J., Vol. 18, No. 7, 1979, pp. 774-778.

${ }^{16}$ Nejad, A.S., and Schetz, J.A., "Effects of Properties and Locations in the Plume on Droplet Diameter for Injection in a Supersonic Stream," AIAA J., Vol. 21, No. 7, 1983, pp. 956-961.

${ }^{17} \mathrm{Nejad}, \mathrm{A} . S$. , and Schetz, J.A., "Effects of Viscosity and Surface Tension on a Jet Plume in Supersonic Cross-Flow, AIAA J., Vol. 22, No. 4, 1984, pp. 458-459.
${ }^{18}$ Less, D.M., and Schetz, J.A., "Transient Behavior of Liquid Jets Injected Normal to a HighVelocity Gas Stream," AIAA J., Vol. 24, No. 12, 1986, pp. 1979-1985.

${ }^{19}$ Kitamura, Y., and Takahashi, T., "Stability of a Liquid Jet in Air Flow Normal to the Jet Axis," J. Chem. Engr. Japan, Vol. 9, No. 4, 1976, pp. 282-286.

${ }^{20}$ Nguyen, T.T., and Karagozian, A.R., "Liquid Fuel Jet in a Subsonic Crossflow," J. Prop. Power, Vol. 8, No. 1, 1992, pp. 21-29.

${ }^{21}$ Karagozian, A.R., "An Analytical Model for the Vorticity Associated with a Transverse Jet," AIAA J., Vol. 24, No. 3, 1986, pp. 429-436.

${ }^{22}$ Higuera, F.J., and Martinez, M., "An Incompressible Jet in a Weak Crossflow," J. Fluid Mech., Vol. 249, 1993, pp. 73-97.

${ }^{23}$ Lienhard, J.H., "Velocity Coefficients for Free Jets from Sharp-Edged Orifices," J. Fluids Engrg., Vol. 106, 1984, pp. 13-17.

${ }^{24}$ Dai, Z., Chou, W.-H., and Faeth, G.M., "Drop Formation Due to Turbulent Primary Breakup at the Free Surface of Plane Liquid Wall Jets," Phys. Fluids, submitted.

${ }^{25}$ Lange, N.A., Handbook of Chemistry, 8th ed., Handbook Publishers, Inc., Sandusky, Ohio, 1952, pp.1134 and 1709.

${ }^{26} \mathrm{Hinze}$ J.O., "Fundamentals of the Hydrodynamic Mechanism of Splitting in Dispersion Processes," AIChE J., Vol. 1, No. 3, 1955, pp. 289-295.

${ }^{27}$ Ranger, A.A., and Nicholls, J.A., "The Aerodynamic Shattering of Liquid Drops," AIAA J., Vol. 7, No. 2, 1969, pp.285-290. 
Table 1. Properties of the test fluids. ${ }^{2}$

\begin{tabular}{llll}
\hline Liquids $^{\mathrm{b}}$ & $\begin{array}{l}\text { Density } \\
\left(\mathrm{kg} / \mathrm{m}^{3}\right)\end{array}$ & $\begin{array}{l}\text { Viscosity } \\
(\mathrm{g} / \mathrm{m}-\mathrm{s})\end{array}$ & $\begin{array}{l}\text { Surface } \\
\text { Tension } \\
(\mathrm{mN} / \mathrm{m})\end{array}$ \\
& & & \\
\hline
\end{tabular}

$\begin{array}{lrrr}\text { Water } & 996 & 0.82 & 70.9 \\ \text { Ethyl Alcohol } & 806 & 1.23 & 22.2 \\ \text { Glycerol (66\%) } & 1172 & 16.4 & 60.7 \\ \text { Glycerol (79\%) } & 1205 & 37.3 & 59.8 \\ \text { Glycerol (84\%) } & 1219 & 80.4 & 63.0\end{array}$

${ }^{2}$ Measured at normal temperature and pressure: $297 \pm 3 \mathrm{~K}$ and $97 \mathrm{kPa}$. Ambient air properties: density of $1.18 \mathrm{~kg} / \mathrm{m}^{3}$ and viscosity of 0.01846 $\mathrm{g} / \mathrm{m}-\mathrm{s}$.

${ }^{b}$ Numbers in parenthesis denote concentration of glycerin by mass.

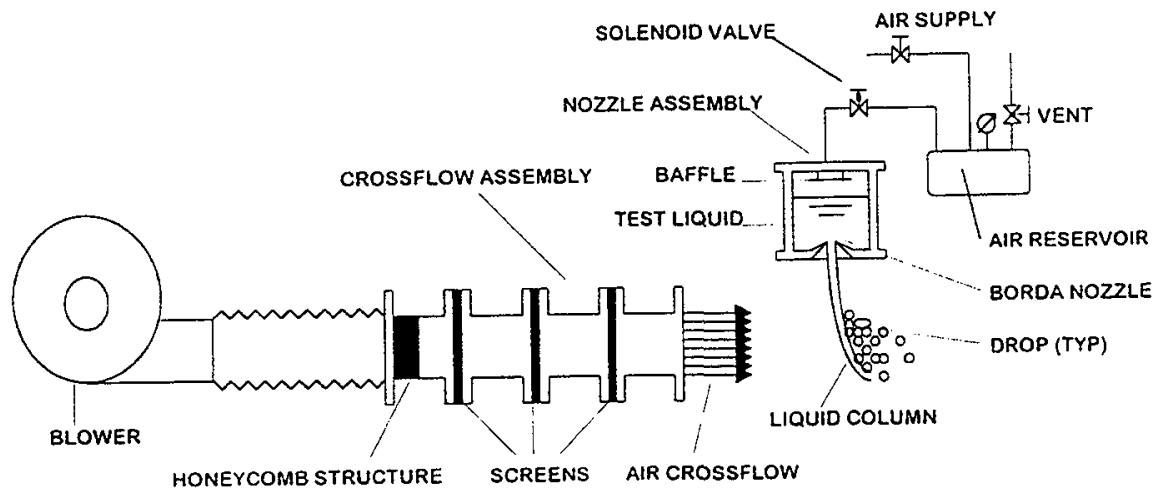

Fig. 1 Sketch of the test apparatus. 


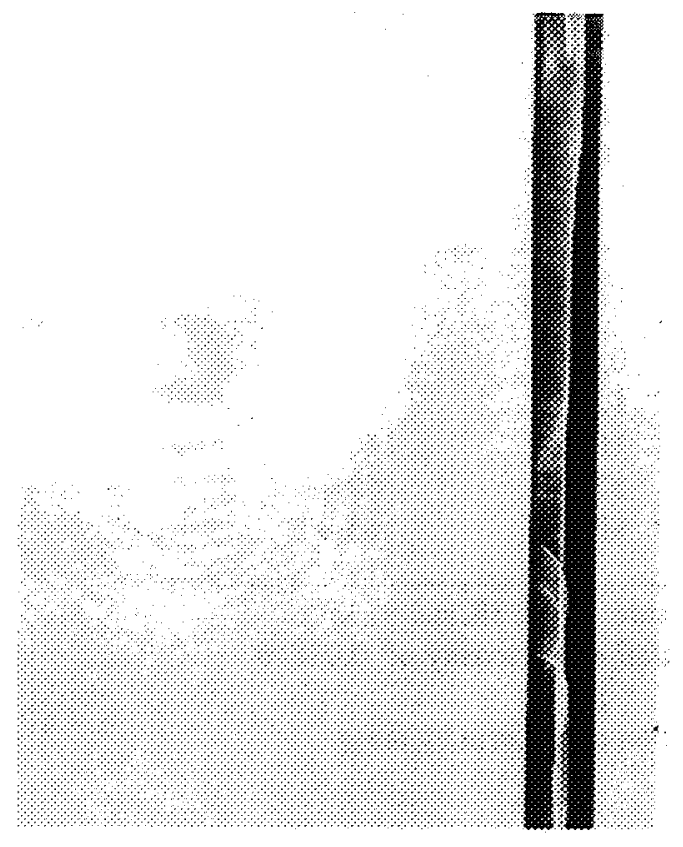

Fig. 2 Pulsed shadowgraph photograph of a nonturbulent round liquid jet in still air at normal temperature and pressure (water, $d_{0}=3.2 \mathrm{~mm}, \mathrm{We}=$ $0, \mathrm{Oh}=0.0017$ and $\mathrm{q}=\infty)$.

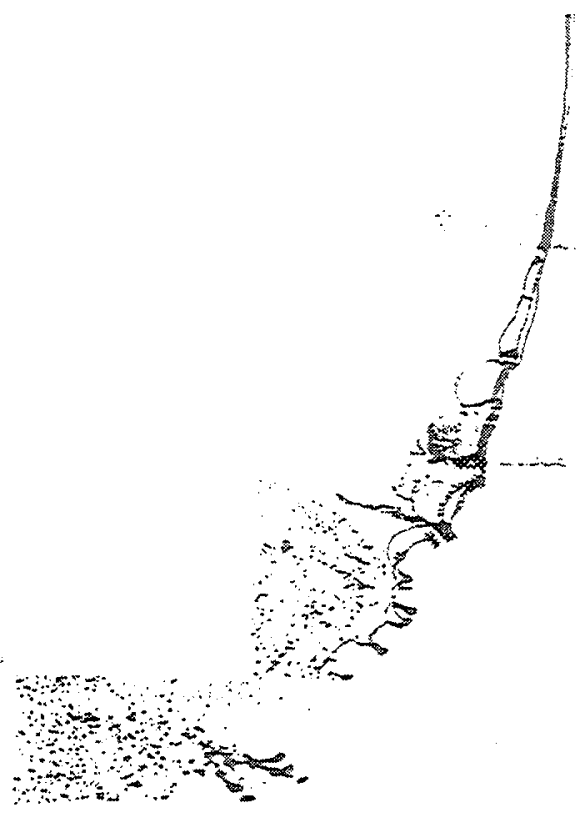

Fig. 3 Pulsed shadowgraph photograph of a nonturbulent round liquid jet in an air crossflow at normal temperature and pressure in the bag breakup regime (water, $d_{o}=1.7 \mathrm{~mm}, \mathrm{We}=6.8, \mathrm{Oh}=$ 0.0024 and $q=242$ ).

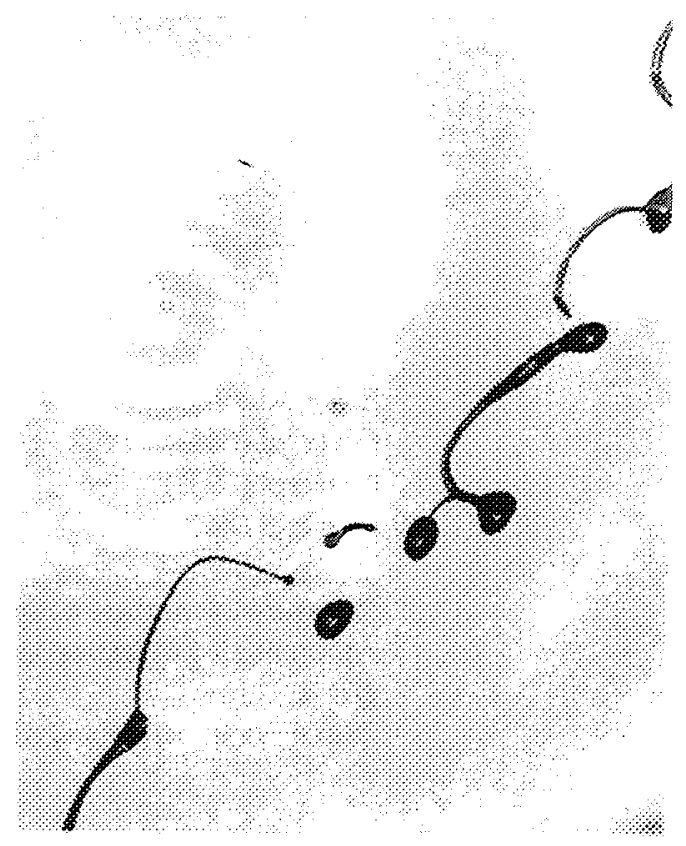

Fig. 4 Pulsed shadowgraph photograph of a nonturbulent round liquid jet in an air crossflow at normal temperature and pressure in the liquid column breakup regime (glycerol $(79 \%), d_{0}=0.8$ $\mathrm{mm}, \mathrm{We}=4.0, \mathrm{Oh}=0.155$ and $\mathrm{q}=562$ ).

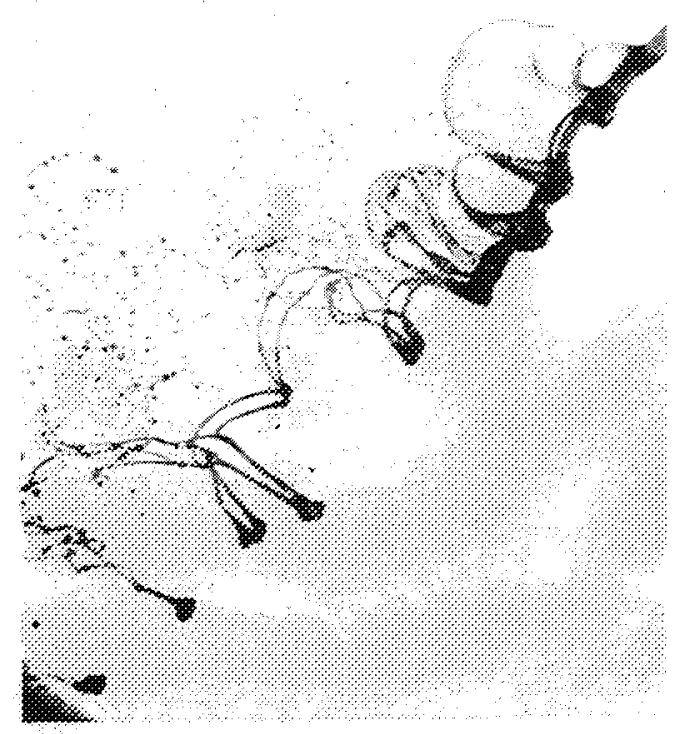

Fig. 5 Pulsed shadowgraph photograph of a nonturbulent round liquid jet in an air crossflow at normal temperature and pressure in the bag breakup regime (glycerol $(84 \%), \mathrm{d}_{0}=0.8 \mathrm{~mm}, \mathrm{We}=7.0$, $\mathrm{Oh}=0.320$ and $\mathrm{q}=308$ ). 


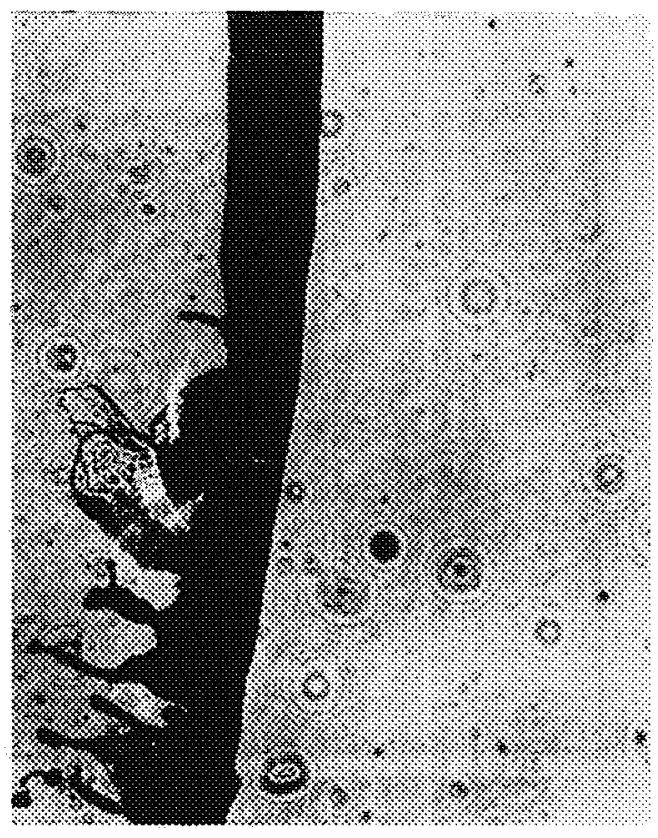

Fig. 6 Pulsed shadowgraph photograph of a nonturbulent round liquid jet in an air crossflow at normal temperature and pressure in the bag/shear breakup regime (water, $\mathrm{d}_{\mathrm{o}}=6.4 \mathrm{~mm}, \mathrm{We}=61, \mathrm{Oh}$ $=0.0012$ and $q=102$ ).

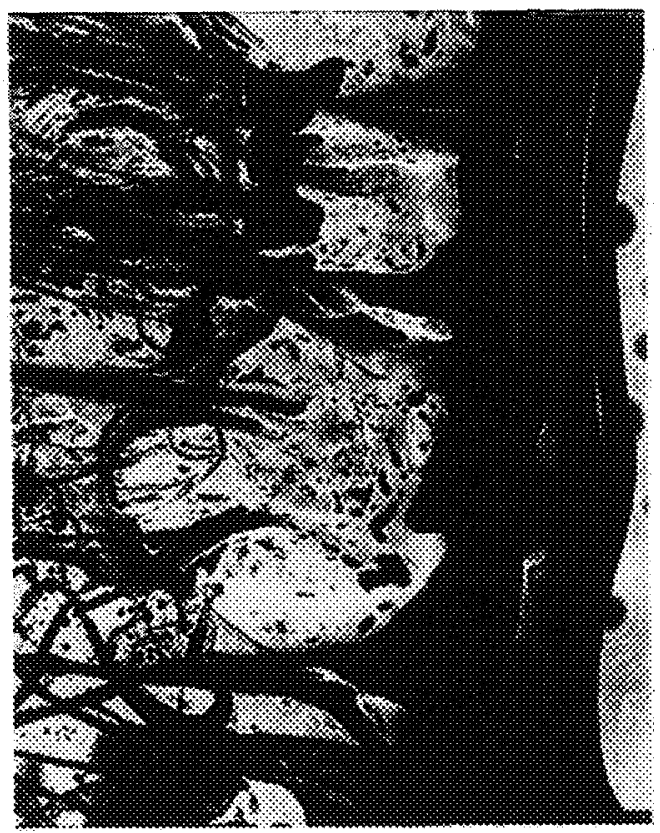

Fig. 8 Pulsed shadowgraph photograph of a nonturbulent round liquid jet in an air crossflow at normal temperature and pressure approaching the long-ligament bag breakup regime (glycerol (79\%), $\mathrm{d}_{\mathrm{o}}=6.4 \mathrm{~mm}, \mathrm{We}=44, \mathrm{Oh}=0.055$ and $\mathrm{q}=220$ ).

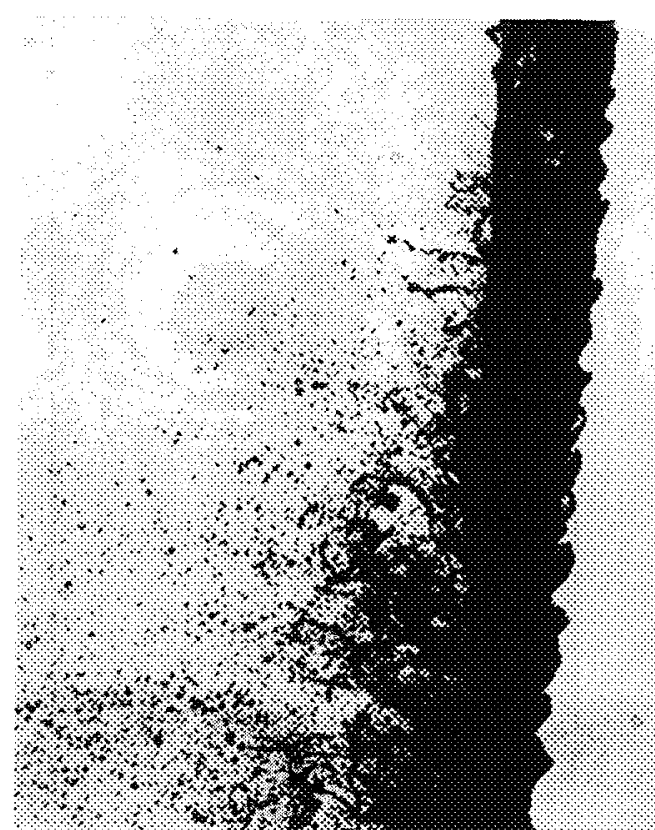

Fig. 7 Pulsed shadowgraph photograph of a nonturbulent round liquid jet in an air crossflow at normal temperature and pressure in the shear breakup regime (ethyl alcohol, $\mathrm{d}_{\mathrm{o}}=7 \mathrm{~mm}, \mathrm{We}=$ $209, \mathrm{Oh}=0.0035$ and $\mathrm{q}=169$ ).

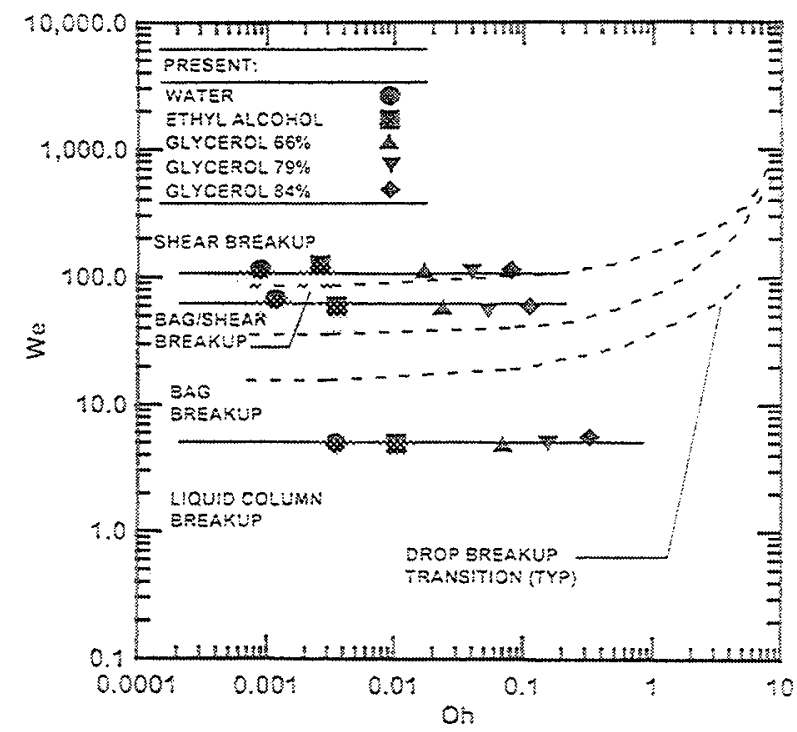

Fig. 9 Breakup regime map for primary breakup of nonturbulent round liquid jets in gaseous crossflows (We $<200$, Oh $<0.4$ and $\mathrm{q}<8000$ ) and for secondary breakup of drops (We $<1000$ and $\mathrm{Oh}$ $<10$ ) Results for secondary breakup of drops from Hsiang and Faeth..$^{5-7}$ 


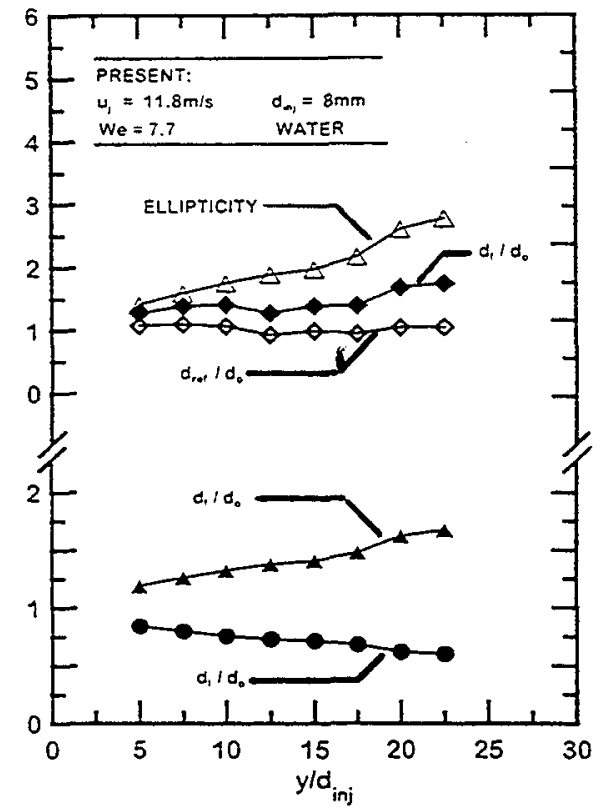

Fig. 10 Deformation of a nonturbulent round liquid jet in an air crossflow at normal temperature and pressure prior to primary breakup (water, $\mathrm{d}_{\mathrm{o}}=$ $6.4 \mathrm{~mm}, \mathrm{We}=7.8, \mathrm{Oh}=0.0012$ and $\mathrm{q}=1590$ ).

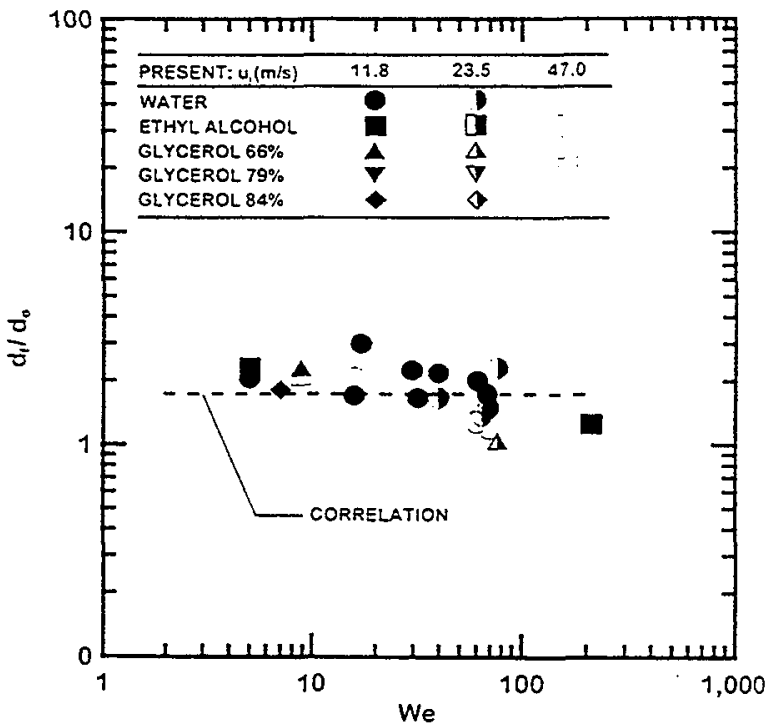

Fig. 11 Maximum deformation at the onset of breakup as a function of Weber number for nonturbulent round liquid jets in an air crossflow at normal temperature and pressure.

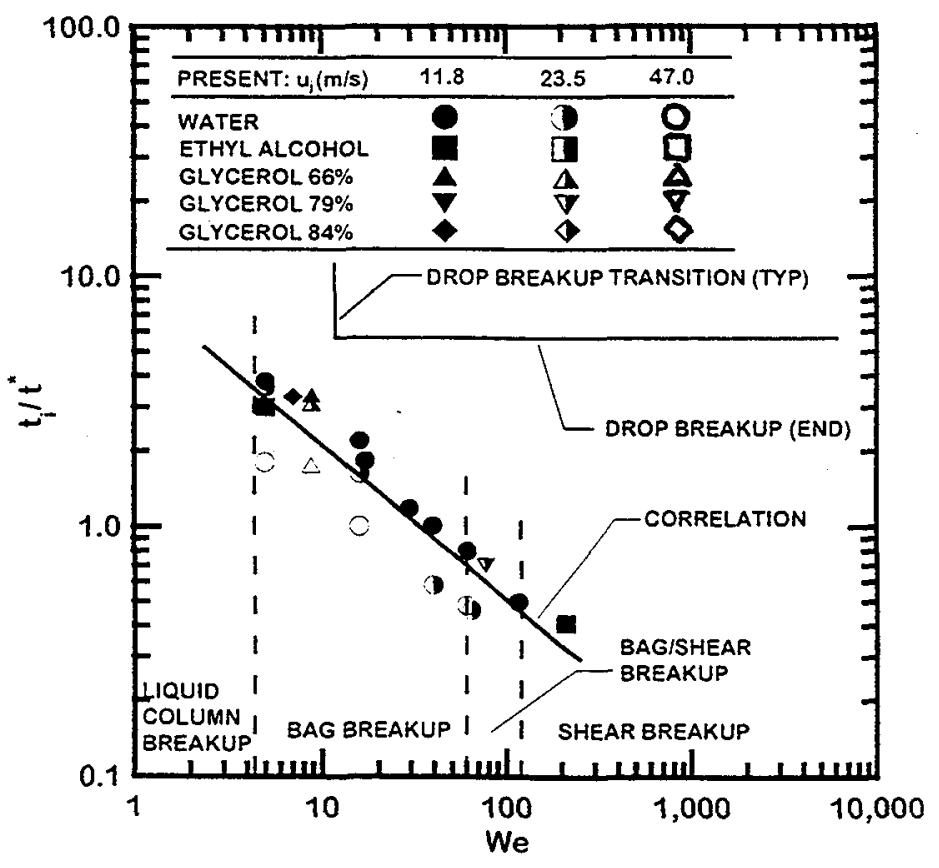

Fig. 12 Time of the onset of breakup as a function of Weber number for nonturbulent round liquid jets an air crossflow at normal temperature and pressure and for completing the secondary breakup of drops. Results for the secondary breakup of drops from Hsiang and Faeth. ${ }^{5-7}$ 


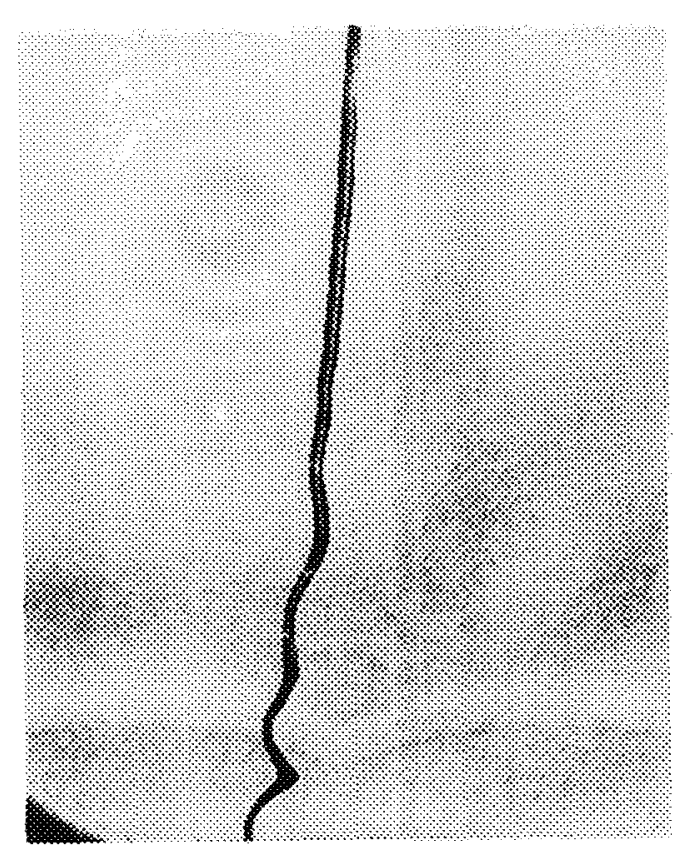

Fig. 13 Pulsed shadowgraph photograph of liquid column waves for a nonturbulent round liquid jet in an air crossflow at normal temperature and pressure (ethyl alcohol, $\mathrm{d}_{0}=0.8 \mathrm{~mm}, \mathrm{We}=4.0, \mathrm{Oh}=$ 0.0103 and $q=815$ ).

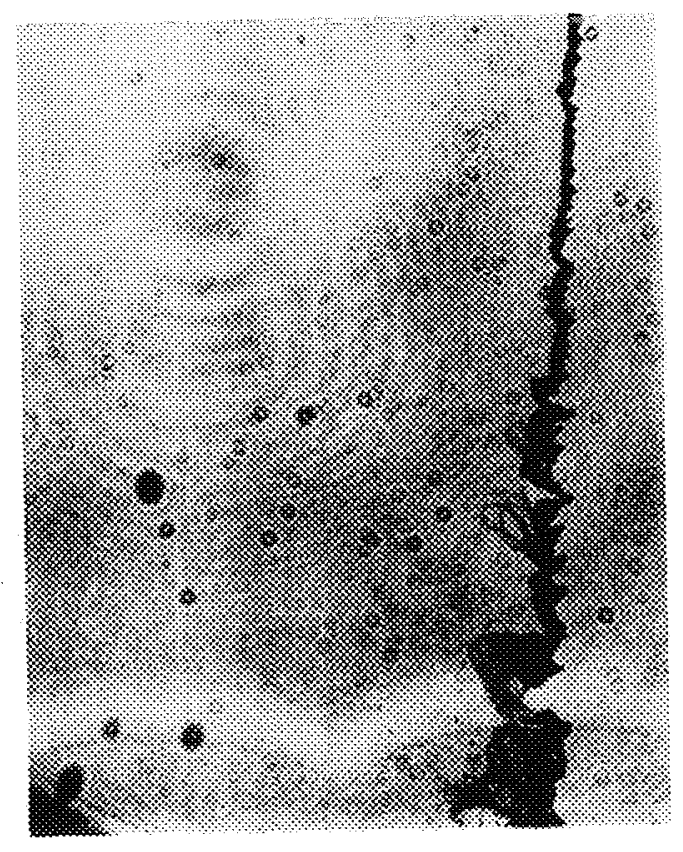

Fig. 14 Pulsed shadowgraph photograph of liquid surface waves for a nonturbulent round liquid jet in an air crossflow at normal temperature and pressure (glycerol $(66 \%), \mathrm{d}_{0}=0.8 \mathrm{~mm}, \mathrm{We}=8.8, \mathrm{Oh}=$ 0.0687 and $q=292$ ).

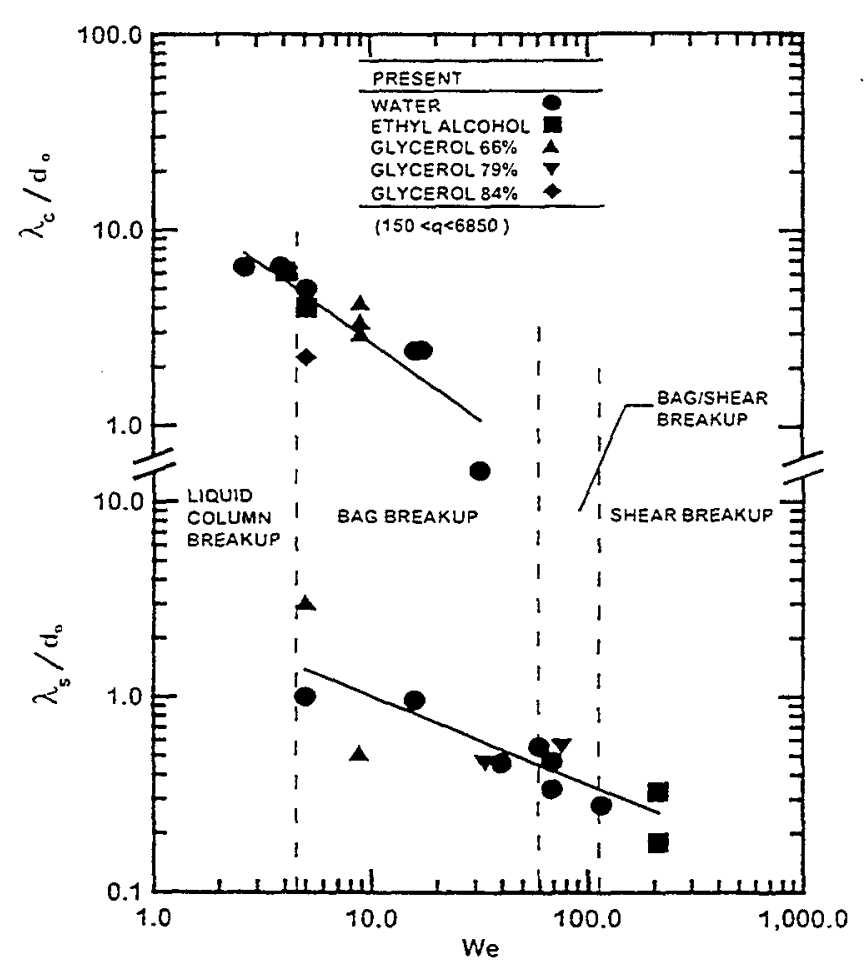

Fig. 15 Liquid column and surface wavelengths of nonturbulent round liquid jets in air crossflows at normal temperature and pressure. 